\title{
A secondary bile acid from microbiota metabolism attenuates ileitis and bile acid reduction in subclinical necrotic enteritis in chickens
}

Mohit Bansal', Ying Fu,2, Bilal Alrubaye ${ }^{1,2}$, Mussie Abraha ${ }^{1}$, Ayidh Almansour ${ }^{1,2}$, Anamika Gupta ${ }^{1}$, Rohana Liyanage ${ }^{3}$, Hong Wang ${ }^{1}$, Billy Hargis ${ }^{1}$ and Xiaolun Sun ${ }^{1,2,3^{*}}$

\begin{abstract}
Background: Clostridium perfringens-induced chicken necrotic enteritis (NE) is responsible for substantial economic losses worldwide annually. Recently, as a result of antibiotic growth promoter prohibition, the prevalence of NE in chickens has reemerged. This study was aimed to reduce NE through titrating dietary deoxycholic acid (DCA) as an effective antimicrobial alternative.

Materials and methods: Day-old broiler chicks were assigned to six groups and fed diets supplemented with 0 (basal diet), $0.8,1.0$ and $1.5 \mathrm{~g} / \mathrm{kg}$ (on top of basal diet) DCA. The birds were challenged with Eimeria maxima (20,000 oocysts/bird) at d 18 and C. perfringens ( $10^{9} \mathrm{CFU} /$ bird per day) at d 23, 24, and 25 to induce NE. The birds were sacrificed at d 26 when ileal tissue and digesta were collected for analyzing histopathology, mRNA accumulation and C. perfringens colonization by real-time PCR, targeted metabolomics of bile acids, fluorescence in situ hybridization (FISH), or terminal deoxynucleotidyl transferase dUTP nick end labeling (TUNEL) assay.

Results: At the cellular level, birds infected with E. maxima and C. perfringens developed subclinical NE and showed shortening villi, crypt hyperplasia and immune cell infiltration in ileum. Dietary DCA alleviated the NE-induced ileal inflammation in a dose-dependent manner compared to NE control birds. Consistent with the increased histopathological scores, subclinical NE birds suffered body weight gain reduction compared to the uninfected birds, an effect attenuated with increased doses of dietary DCA. At the molecular level, the highest dose of DCA at $1.5 \mathrm{~g} / \mathrm{kg}$ reduced C. perfringens luminal colonization compared to NE birds using PCR and FISH. Furthermore, the dietary DCA reduced subclinical NE-induced intestinal inflammatory gene expression and cell apoptosis using PCR and TUNEL assays. Upon further examining ileal bile acid pool through targeted metabolomics, subclinical NE reduced the total bile acid level in ileal digesta compared to uninfected birds. Notably, dietary DCA increased total bile acid and DCA levels in a dose-dependent manner compared to NE birds.

(Continued on next page)
\end{abstract}

\footnotetext{
* Correspondence: xiaoluns@uark.edu

'Center of Excellence for Poultry Science, University of Arkansas, $1260 \mathrm{~W}$

Maple St. O409, Fayetteville, AR 72701, USA

${ }^{2}$ CEMB, University of Arkansas, Fayetteville, AR 72701, USA

Full list of author information is available at the end of the article
}

(c) The Author(s). 2020 Open Access This article is licensed under a Creative Commons Attribution 4.0 International License, which permits use, sharing, adaptation, distribution and reproduction in any medium or format, as long as you give appropriate credit to the original author(s) and the source, provide a link to the Creative Commons licence, and indicate if changes were made. The images or other third party material in this article are included in the article's Creative Commons licence, unless indicated otherwise in a credit line to the material. If material is not included in the article's Creative Commons licence and your intended use is not permitted by statutory regulation or exceeds the permitted use, you will need to obtain permission directly from the copyright holder. To view a copy of this licence, visit http://creativecommons.org/licenses/by/4.0/ The Creative Commons Public Domain Dedication waiver (http://creativecommons.org/publicdomain/zero/1.0/) applies to the data made available in this article, unless otherwise stated in a credit line to the data. 
(Continued from previous page)

Conclusion: These results indicate that DCA attenuates NE-induced intestinal inflammation and bile acid reduction and could be an effective antimicrobial alternative against the intestinal disease.

Keywords: Bile acid, Chicken, Clostridium perfringens, Deoxycholic acid, Intestinal inflammation, Necrotic enteritis

\section{Background}

$\mathrm{NE}$ is an intestinal disease in chickens. The main causative pathogen of NE is a spore-forming, anaerobic, and gram-positive bacterium Clostridium perfringens and NE is often associated with the predisposing factor of Eimeria maxima and/or E. acervulina infection. Birds suffering acute NE show sudden death of up to $50 \%$ mortality [1]; however, the more common form of NE is subclinical with neither obvious clinical signs present nor peak mortality. Antimicrobials have been used to prevent coccidiosis and NE [2], but the growing concerns on antimicrobial resistance promotes the restriction of their usage $[2,3]$.

The practice to withdraw antimicrobials in poultry production is facing a number of challenges. Among them is the elevated productivity loss from chicken diseases, such as NE. Recently, the reemergence of NE has costed poultry industry $\$ 6$ billion annually worldwide [4]. Severely ill and deceased NE birds show fragile intestines ballooned with gas and a foul-smelling brown fluid [5]. The severity of $\mathrm{NE}$ is often diagnosed using gross lesion scores [6]. At the cellular level, the intestinal tract of acute NE birds displays severe small intestinal inflammation from an infiltration of massive immune cells into the lamina propria, villus epithelial line necrosis and crypt hyperplasia [7-9]. Progress has been made in understanding the risk factors influencing the outcome of NE such as $C$. perfringens virulence, coccidiosis and feed [10]. However, little progress has been made in developing strategies for antibiotic alternatives to prevent and treat NE.

C. perfringens colonizes the intestinal tract among normal chicken microbiota. The presence of high doses of $C$. perfringens in the intestine does not sufficiently induce NE and the count alone does not associate with $\mathrm{NE}$ [11-13]; therefore, the development of NE is dependent on C. perfringens toxins and predisposing factors, such as coccidiosis, feed and microbiota. Coccidiosis is the most comment predisposing factors in field $\mathrm{NE}$ cases [14]. It is well known that different strains of C. perfringens produce a variety of toxins such as alpha (CPA, type A), beta toxin 2 (CPB2), enterotoxin (CPE), necrotic enteritis B-like toxin (NetB), TpeL and others [15]. Among them, C. perfringens isolated from NE birds are mainly type A strains. Researchers in Australia have reported that NetB induced NE in chickens, but not CPA [16]. However, C. perfringens isolated from clinical NE birds isn't always associated with virulent genes of
netB, $c p b 2$, tpeL or cpe $[17,18]$. Thus, more researches are needed to investigate the relationship between $\mathrm{NE}$ and $C$. perfringens virulent factors, particularly in the presence of predisposing factor of coccidiosis.

Human and animal intestines harbor up to trillions of microbes which regulate various host functions such as the intestinal barrier, nutrition and immune homeostasis [19-22]. Microbiota composition is altered in NE birds compared to uninfected birds [23], which may lead to bile acid changes in the host. At the gut level, microbiota reconstitution by transplantation has shown tremendous success against recurrent Clostridium difficile infection [24]. The reduction of $C$. difficile infection is attributed to the secondary bile acids produced by the transplanted normal microbiota member C. scindens [25]. Bile acids are synthesized and conjugated in the liver, including tauroor glycol-cholic acids (TCA or GCA) and tauro- or glycolchenodeoxycholic acids (TCDCA or GCDCA). After the conjugated bile acids are released, they are metabolized in the intestine by various gut microbiota through deconjugation (e.g. CA or CDCA) and transformed into secondary bile acids, such as deoxycholic acid (DCA), lithocholic acid (LCA) or ursodeoxycholic acid (UDCA) [26]. The majority of bile acids (>95\%) are effectively absorbed in the intestine through enterohepatic circulation/cycle [26]. Bile acids have been reported in association with a variety of chronic diseases [27, 28], but new evidence sheds light on the beneficial property of secondary bile acids in health and disease, such as increasing gut motility [29] and reducing $C$. difficile infection [25]. It remains largely unknown how dietary bile acids modulate intestinal bile acid pool in health and disease.

Anaerobic microbiota and its metabolite DCA prevent Campylobacter jejuni-induced intestinal inflammation [30] in mice and bacterial colonization in chickens [31]. Recently, we found that E. maxima and C. perfringens induced acute NE, body weight loss and intestinal inflammation, which was effectively attenuated by dietary DCA at $1.5 \mathrm{~g} / \mathrm{kg}$ [9]. We reasoned that dietary DCA would modulate NE in a dose response manner. To address this possibility, we conducted a following up chicken experiment. In the current experiment, different dietary doses of DCA were used to prevent subclinical $\mathrm{NE}$ and to investigate the impact of dietary DCA on intestinal bile acid pool. The results from this study will help the understanding of NE pathogenesis and assist in developing new antimicrobial alternatives against NE. 


\section{Materials and methods Chicken experiment}

The experiment was conducted at the Poultry Health Laboratory of University of Arkansas at Fayetteville. All the experimental procedures were approved by the Animal Care and Use Committee of the University of Arkansas. A total of 96 male, 1-day-old broiler chicks were obtained from Cobb-Vantress (Siloam Springs, AR). Upon arrival, broiler chicks were neck-tagged, individually weighed and randomly assigned to one of 6 floor pens in a controlled age appropriate environment. Birds were fed with $0,0.8$, 1.0 and $1.5 \mathrm{~g} / \mathrm{kg}$ DCA (Alfa Aesar, MA, US) on top of basal diets from d 0 to 26 . The basal diets were a cornsoybean meal-based starter diet during d 0 to10 and a grower diet during $\mathrm{d} 11$ to 26 [9]. Birds were infected with 20,000 sporulated E. maxima M6 oocysts at d 18 [9]. E. maxima used was propagated and sporulated $1 \mathrm{yr}$ before the chicken experiment. The birds were then infected with C. perfringens $[32,33]$ with $10^{9} \mathrm{CFU} /$ bird at $\mathrm{d} 23,24$ and 25 [9]. This $C$. perfringens isolate was confirmed to be net $B$ and cpe positive by PCR. Chicken body weight was measured at d 0,18, 23 and 26. Birds were sacrificed at $d$ 26 to collect ileal tissue and digesta for analysis of histopathology, bacterial colonization and inflammation. In a separate trial, 16 birds/pen were challenged as before and feed intake was calculated.

\section{Histopathology analysis of intestinal inflammation}

Distal jejunal $(2 \mathrm{~cm})$ and proximal ileal tissue $(8 \mathrm{~cm})$ adjacent to Meckel's diverticulum was removed, Swissrolled, and fixed in $10 \%$ phosphate-buffed formalin $(\mathrm{pH}$ 7.4) overnight at $4{ }^{\circ} \mathrm{C}$. Tissue samples were then embedded in paraffin and sections $(5 \mu \mathrm{m})$ were cut, processed and stained with H\&E at the Histology Laboratory in the Department of Poultry Science at the University of Arkansas in Fayetteville, Arkansas. Each Swiss-roll slide at ileum was evaluated for ileal inflammation based on the four-characteristic NE lesion score system. The four characteristics of NE lesion are level of immune cell infiltration in lamina propria, villus length, crypt hyperplasia and tissue ulceration [9]. Briefly, continuous (decimal) scoring method was used on scale of 0-4 where 0 indicates: no inflammation, villi and crypt intact; score 1: small number infiltration cells in laminar propria of villi and crypts or villi minimally shortened; score 2: more extensive infiltration cells in laminar propria of villi and crypts, villi shortened $>1 / 4$ and edema, or crypt hyperplasia; score 3: pronounced infiltration cells in laminar propria of villi, crypts, submucosa, and muscularis, villi shortened $>1 / 2$ and edema, or crypt hyperplasia and regeneration; and score 4: necrosis, villus diffuse, ulcers, crypt abscesses, or transmural inflammation (may extend to serosa). Images of representative histopathology were acquired using a Nikon TS2 fluorescent microscope.
C. perfringens colonization in ileal lumen using real-time PCR and fluorescence in situ hybridization (FISH)

Ileal digesta samples were collected from birds scarified on d 26. Total DNA was extracted using phenol/chloroform method as previously described [9]. Real-time PCR was performed to quantify the colonization level of C. perfringens in ileal digesta targeting $16 \mathrm{~S}$ rDNA and using a bacterial standard as described before [9]. FISH was performed to visualize $C$. perfringens intestinal colonization using histology slides as described before [9].

\section{Host inflammatory response and bacterial gene using real-time PCR and terminal deoxynucleotidyl transferase dUTP nick end labeling (TUNEL) assay}

The host immune response of inflammatory gene expression was evaluated in ileal tissue samples. The total RNA was extracted from ileal tissue samples of birds sacrificed at d 26 using TRIzol as described before [9, 34] and cDNA was prepared using M-MLV (NE Biolab). The accumulation of proinflammatory genes of Infy, Mmp9, Il17a, Il22 and Il23 in ileum tissue were determined using SYBR Green PCR Master mix (Bio-Rad) on a Bio-Rad 384-well RealTime PCR System. The primer sequences of each gene were described before [9] and here: Il22_forward: 5'-CTGCCC ATAGCTGCAGTACA-3'; Il22_reverse: 5' - 3'; Il23_forward: 5' -ATGCATTGCGATGTCTGAAG-3'; Il23_reverse: 5'-ACTTGGGTGCTTCCAAGATG-3'. Gene expression of fold changes in uninfected birds was calculated using $\Delta \Delta C$ t method [35] and Gapdh was used as the internal control.

C. perfringens virulent DNA genes and $16 \mathrm{~S}$ (internal control) were detected using real-time PCR and the primer sequences were: netB_forward: GGAAAAATGA AATGGCCTGA; netB_reverse: GCACCAGCAGTTTT TCCTTC; $c p e$ forward: CAACTGCTGGTCCAAATGAA; cpe_reverse: GCATCTTTCGCCAGTTTCAA; 16S_ forward: AGGAGCAATCCGCTATGAGA; 16S_reverse: GTGCAATATTCCCCACTGCT.

\section{Quantification of ileal bile acids using targeted metabolomics}

The ileal digesta was collected and the bile acid composition was analyzed using multiple reaction monitoring (MRM) mass spectrometry in Statewide Mass Spectrometry Facility at the University of Arkansas in Fayetteville, Arkansas. Five or six ileal digesta samples from each group were used for extraction of bile acids. The Intestinal bile acids were extracted using chloroform/methanol. To identify and quantify bile acids, MRM methods have been developed for tauro- and glyco-cholic acid (TCA/GCA), tauro- and glycochenodeoxycholic acid (TCDCA/GCDCA), cholic acid (CA), deoxycholic acid (DCA), lithocholic acid (LCA), ursodeoxycholic acid (UDCA) isotopically labeled (some were with deuteriums and some were with five deuteriums) and 
unlabeled standards. The methods were fully optimized using data acquisition software attached to Shimadzu UPLC-20A/LC-30A coupled with Shimadzu 8050 triple quadrupole mass spectrometer at their respective retention times. Once the methods were optimized, the samples with isotopically labeled internal standards were analyzed along with standards of varying concentrations. Calibration curves with internal standards were used to estimate bile acid concentration. The use of the internal standard accounts for the sample loss during the extraction procedure. LC-MS/ MS analysis was performed in the negative-ion mode. Methodology is similar to what has been described before [36]. LC separation was performed using a C18 column. The flow rate was $0.3 \mathrm{~mL} / \mathrm{min}$ and sample volumes of $1 \mu \mathrm{L}$ were injected. The three most intense multiple reaction monitoring (MRM) fragments for each bile acid standard were fully optimized at their respective retention time window using Shimadzu Labsolution software as described before. From the three MRM channels, the MRM event corresponding to the most intense fragment ion was used as the "quant" ion, and the other two fragment ions were used as reference ions for identification purposes. Reference ion ratios were calculated compared to the "quant" ion, and unknown samples were required to meet within $30 \%$ of the reference ion ratio of the standard.

\section{Statistical analysis}

Differences between treatments were analyzed using One-way ANOVA followed by Dunnette's multiple comparison test using Prism 7.0 software. Data were also analyzed using the nonparametric Mann-Whitney $U$ test using Prism 7.0 software. Values are shown as a mean of samples in the treatment \pm standard error of the mean as indicated. Experiments were considered statistically significant if $P$ values were $<0.05$. Regression models were constructed to generate the correlations between dietary DCA levels and host responses (body weight gain, histopathological score and bile acids). $\mathrm{R}^{2}$ was used to indicate the strength of correlation.

\section{Results}

DCA attenuated subclinical NE-induced ileal inflammation In contrast to the previous experiment, NE birds in current experiment showed mild diarrhea (wet bedding) and no mortality. We then examined NE disease development at the cellular level using histopathology analysis. Consistent with the mild subclinical signs, ileum in NE birds showed moderate ileitis with mild crypt hyperplasia, villus shortening and infiltration of immune cells in lamina propria (Fig. 1a). Significantly, dietary DCA at $0.8,1.0$, and $1.5 \mathrm{~g} / \mathrm{kg}$ reduced the intestinal inflammation and histopathological scores by $23 \%, 48 \%$ and $61 \%$ respectively compared to NE birds (Fig. 1a and b). To develop a deeper understanding about the relationship between dietary DCA levels and NE ileitis, a dose response curve was generated. Notably, the lowest dose of DCA to attenuate subclinical NE was at $0.5682 \mathrm{~g} / \mathrm{kg}$ (Fig. 1c), while DCA at $1.359 \mathrm{~g} / \mathrm{kg}$ would reduce subclinical NE to the lowest score of 2.67.

Because DCA at $1.5 \mathrm{~g} / \mathrm{kg}$ exhibited best potential to reduce NE, we focused our attention on DCA at that dose against host inflammatory mediator mRNA expression using real-time PCR. NE induced significantly higher accumulation of ileal inflammatory mRNA mediators of Infy, Mmp9, Il17a, Il22, and Il23 by 3.32, 10.6, 6.02, 3.56 and 4.16 folds, respectively, compared to uninfected birds (Fig. 2a). Remarkably, DCA at $1.5 \mathrm{~g} / \mathrm{kg}$ attenuated the NE-induced inflammatory mediators of Infy, Mmp9, $I l-17 a, I l-22$, and $I l-23$ by $76 \%, 81 \%, 76 \%, 79 \%$, and $58 \%$, respectively. To further understand how DCA against subclinical NE, TUNEL assay was performed to examine cell death. Consistent with the inflammatory mediator gene expression, NE induced substantial epithelial (arrow) and lamina propria immune cell death (arrow head) in ileal villi of the subclinical NE birds (Fig. 2b), whereas dietary DCA at $1.5 \mathrm{~g} / \mathrm{kg}$ attenuated the effect.

\section{DCA reduced subclinical NE-induced body weight gain loss}

We then evaluated how subclinical NE impacted birds' daily body weight gain. Birds grew comparably between different groups during the uninfected phase of $\mathrm{d} 0$ to 18 (Fig. 3a). Interestingly, there was a minimal difference of body weight gain between uninfected and E. maxima-infected birds during E. maxima phase (EM) from d 18 to 23 , suggesting a mild coccidiosis from the E. maxima infection in this experiment. Notably, body weight gain in NE birds was reduced by $28 \%$ compared to uninfected birds during NE phase from d 23 to 26 (155 vs. $110.7 \mathrm{~g} / \mathrm{d}$, $P<0.05)$; an effect attenuated by birds fed with 1.0 and $1.5 \mathrm{~g} / \mathrm{kg}$ DCA diet (110.7 vs. 150 and $148 \mathrm{~g} / \mathrm{d}, P<0.05)$. To have further understanding of the relationship between dietary DCA levels and body weight gain in NE, a dose response curve was generated. The lowest dose of DCA to increase body weight gain compared to NE was $0.68 \mathrm{~g} / \mathrm{kg}$ (Fig. 3b), while DCA at $1.262 \mathrm{~g} / \mathrm{kg}$ would increase body weight gain by $75.266 \mathrm{~g} / \mathrm{d}$.

\section{DCA reduced $C$. perfringens colonization in ileum}

Next, we quantified C. perfringens colonization using realtime PCR. Notably, $C$. perfringens in ileum colonized more than 2 logs in NE birds compared to uninfected birds (Fig. 4a). Apart from a previous report of DCA against acute NE [9], DCA at $1.5 \mathrm{~g} / \mathrm{kg}$ reduced $C$. perfringens colonization by more than 5 folds compared to NE birds. To reveal the spatial distribution of $C$. perfringens, FISH was also performed on the histology tissue slides to reveal the ileal distribution of $C$. perfringens. Interestingly, C. perfringens in uninfected birds was barely detected in the 


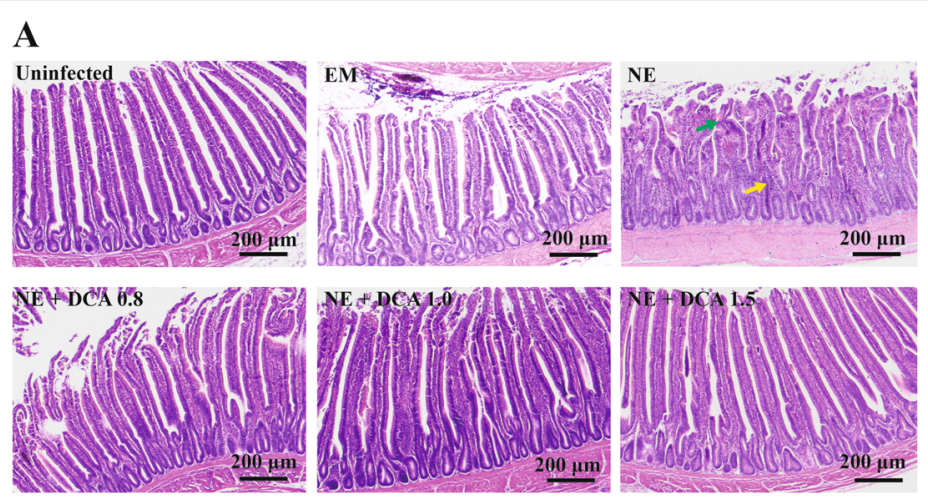

B

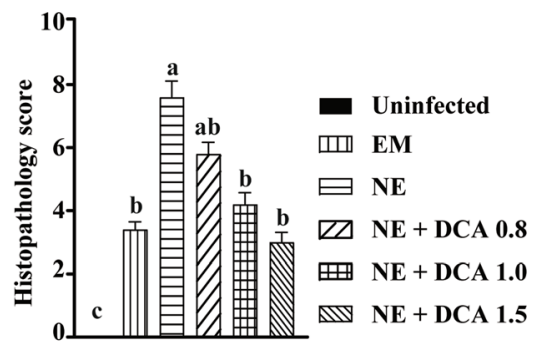

C

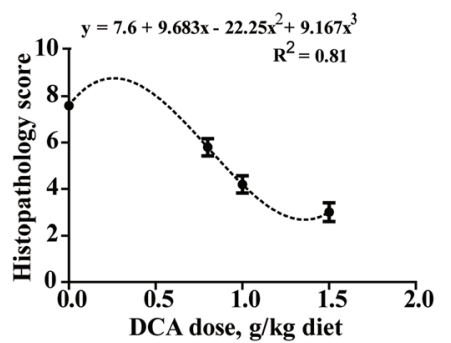

Fig. 1 DCA prevented subclinical NE-induced ileal inflammation. Cohorts of 16 birds were fed diets supplemented with $0,0.8,1.0$ and $1.5 \mathrm{~g} / \mathrm{kg}$ DCA. Birds were challenged with E. maxima at $d 18$ and C. perfringens at d 23, 24, and 25. Randomly selected five birds from each treatment groups were sacrificed and ileal tissue samples were collected. a Representative images of H\&E-stained ileal tissue sections showing immune cell infiltration (yellow arrow) and blunted villi (green arrow). b Histopathology score of ileal tissue. c Fitting curve for histopathology scores in response to different doses of DCA. All graphs showed mean \pm SEM. Different letters of $a, b$ and $c$ mean $P<0.05$. Results are representative of 3 independent experiments. Scale bar is $200 \mu \mathrm{m}$

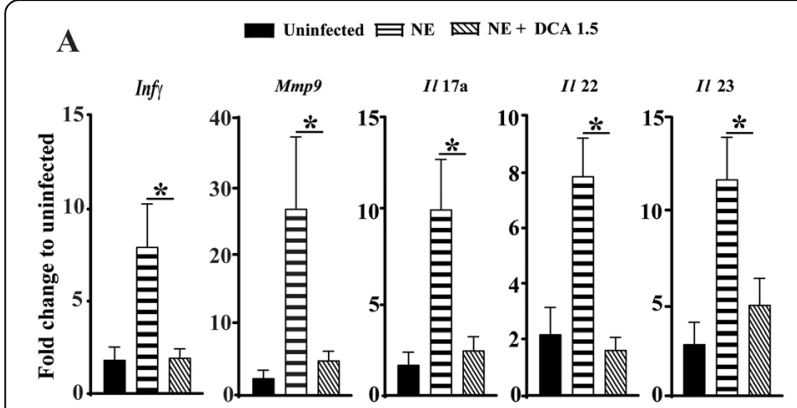

B
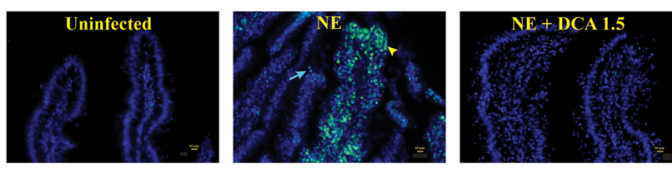

Fig. 2 DCA reduced NE-induced host inflammatory response. Cohorts of broiler chickens were fed with different doses of DCA and infected as in Fig. 1. a lleal Infy, Mmp9, I/17A, I/22 and I/23 mRNA fold changes relative to uninfected birds and normalized to Gapdh. b Representative TUNNEL assay images showing epithelial (Arrow) and immune cell cell apoptosis (arrow head). Scale bar is $10 \mu \mathrm{m}$. All graphs depict mean \pm SEM. * indicates $P<0.05$. Results are representative of 3 independent experiments lumen and tissue of ileum (Fig. 4b), although it was detected in PCR assay. C. perfringens in rod- (arrow head) or spore-shape (arrow) was present in ileal lumen and inside of the villi of subclinical NE birds. C. perfringens was barely visualized in birds fed DCA at $1.5 \mathrm{~g} / \mathrm{kg}$.

\section{Dietary DCA restored NE-induced total bile acid level reduction}

Bile acids in intestinal digesta were quantified using targeted metabolomics. Notably, the level of total bile acids was at 7,638 nmol/g ileal digesta of uninfected birds (Fig. 5a). Proportionally, the bile acid pool was comprised of $58 \%$ conjugated (TCDCA, GCDCA, TCA and GCA) and $42 \%$ deconjugated bile acids (CDCA, CA, DCA, LCA and UDCA) (Fig. 5b). Conjugated and deconjugated primary bile acids (TCDCA, GCDCA, TCA, GCA, CDCA and $\mathrm{CA}$ ) constituted $99.6 \%$ of total bile acids in ileum, whereas secondary bile acids of DCA, LCA and UDCA comprised only $0.4 \%$ of total bile acids.

We then examined the impact of NE and dietary DCA on ileal bile acid pool. NE reduced the level of ileal total bile acids by $67 \%$ compared to uninfected birds (7,638 vs. 2,530 $\mathrm{nmol} / \mathrm{g}$ ), whereas E. maxima infection did not significantly 

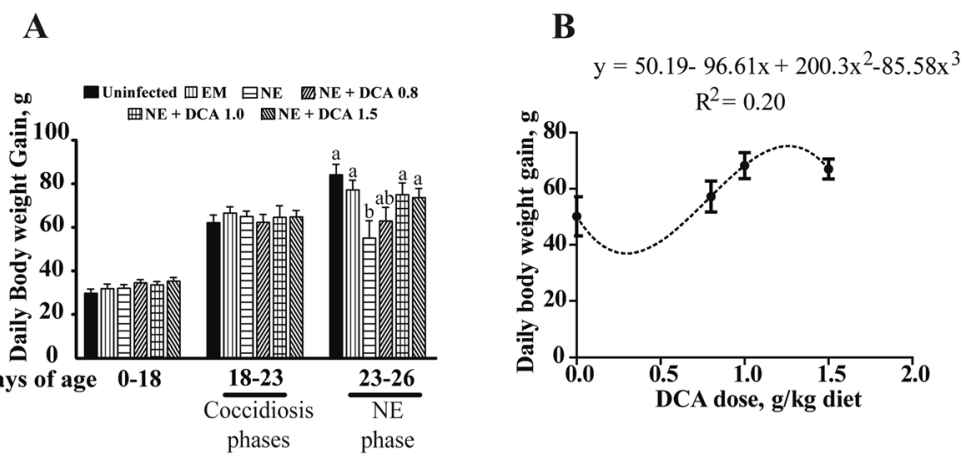

Fig. 3 DCA prevented NE induced body weight gain loss. Cohorts of 16 broiler chicks were fed basal and DCA supplemented diets (0.8, 1.0 and $1.5 \mathrm{~g} / \mathrm{kg}$ ) and infected as in Fig. 1. a Bird body weight gain was measured at $\mathrm{d} 18,23$ and 26. Showed were periodic body weight gain at $\mathrm{d} 0$ to 18,18 to 23 and 23 to 26. b Fitting curve for daily body weight gain at d 23 to 26 in response to different doses of DCA. All graphs showed mean \pm SEM. Different letters of $\mathrm{a}$ and $\mathrm{b}$ mean $P<0.05$. Results are representative of 3 independent experiments

reduce the bile acid level (Fig. 6a). Meanwhile, subclinical $\mathrm{NE}$ infection reduced feed intake during NE phase only by $14 \%$ (Supple Fig. 1). Dietary DCA at $0.8,1.0$, and $1.5 \mathrm{~g} / \mathrm{kg}$ increased the total bile acid levels in a dose dependent manner $(4,000,5,900$, and 7,500 nmol/g, respectively) (Fig. 6b). As a result, the relative proportion of DCA in ileal digesta increased from $0.20 \%$ in NE birds to $31 \%, 63 \%, 74 \%$, respectively, in birds fed with a DCA diet at $0.8,1.0$, and $1.5 \mathrm{~g} / \mathrm{kg}$ (Fig. 6c). To further understand the relationship between dietary DCA supplementation and ileal bile acid composition, we drew three correlation lines (Fig. 6d). From the figure, it was possible to predict ileal bile acid composition based on dietary bile acid supplementation, or vice versa.

\section{Discussion}

In current study, the birds experienced subclinical NE possibly because of mild coccidiosis from infection with old E. maxima. Importantly, DCA prevented the subclinical NE on intestinal inflammation and body weight gain loss in a dose dependent manner. Dietary DCA increased total bile acid level and modulated bile acid composition in ileum. The results from this study indicate that dietary DCA is able to reduce subclinical NE and modulate intestinal bile acid reduction.

Many predisposition factors shape the outbreak and severity of NE, such as coccidiosis, feed and $C$. perfringens virulence [10]. Specifically, E. maxima invades and induces lesions in jejunum and ileum [37], where the NE lesions are often observed [14]. The spatial overlap between $\mathrm{NE}$ - and E. maxima-induced lesions implicate coccidiosis as the most important predisposed factor for the subsequent NE. Consistent with this notion, NE birds in this study experienced mild body weight gain loss during the NE phase after a mild EM phase on body weight gain. This result highlights the important influence of coccidiosis on the outcome of NE. DCA was able to prevent the body weight gain loss in a dose dependent manner, following the construction of the $3^{\text {rd }}$ order polynomial fitting model using dietary DCA levels and body weight gain during NE phase in Fig. 3b. Interestingly, DCA at $1.5 \mathrm{~g} / \mathrm{kg}$

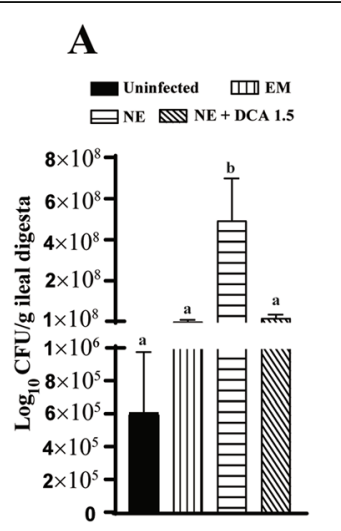

B
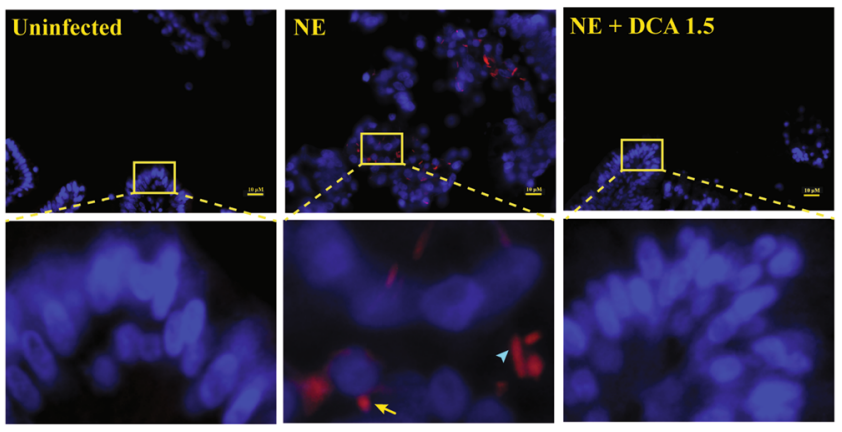

Fig. 4 DCA reduced C. perfringens ileal colonization. Cohorts of broiler chickens were fed with different doses of DCA and infected as in Fig. 1. a C. perfringens was quantified in ileal digesta using PCR. b C. perfringens was visualized in ileal tissue using FISH. All graphs showed mean \pm SEM. Different letters of $\mathrm{a}$ and $\mathrm{b}$ mean $P<0.05$. Results are representative of 3 independent experiments. Scale bar is $10 \mu \mathrm{m}$ 

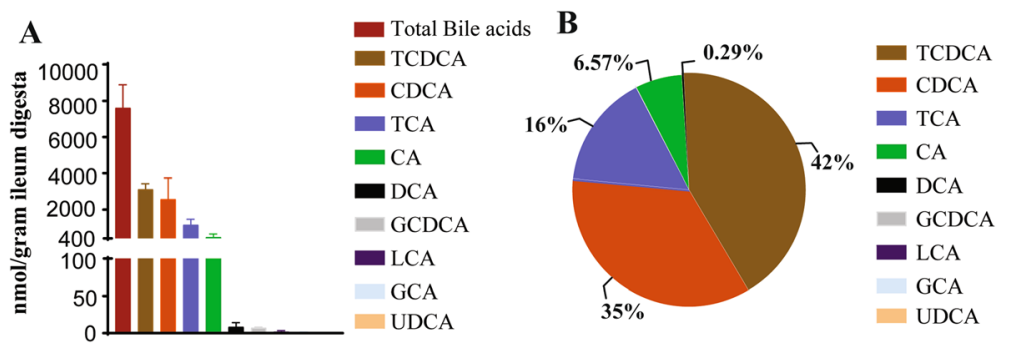

Fig. 5 Composition of bile acids in ileal digesta of healthy birds. Bile acids in ileal digesta of 5 healthy birds were extracted and quantified using targeted metabolomics. a Bile acids quantification in the healthy bird ileum. $\mathbf{b}$ Relative composition of bile acid in the healthy birds. All graphs showed mean \pm SEM. Results are representative of 3 independent experiments

A

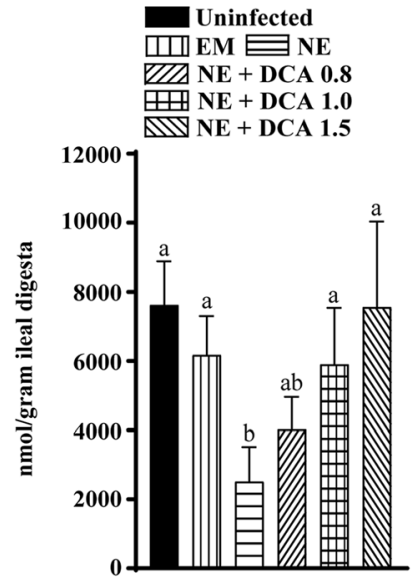

C $\because T C D C A \backsim G C D C A \backsim C D C A \backsim T C A$ $\because \mathrm{GCA} \backsim \mathrm{CA} \backsim \mathrm{DCA} \backsim \mathrm{LCA}=\mathrm{UDCA}$

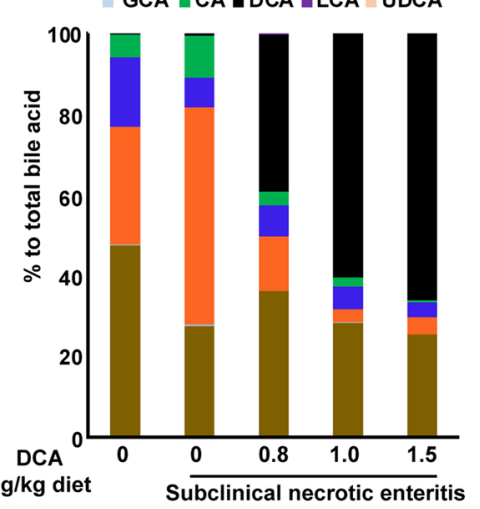

B

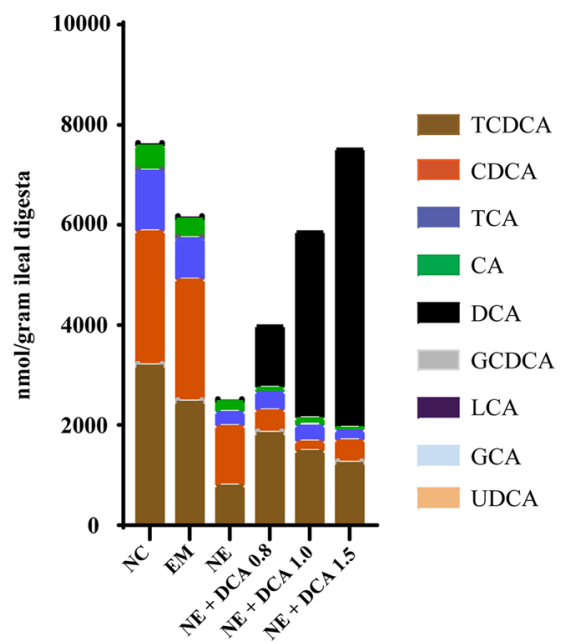

D

For DCA: $y=0.6312-109.7 x+303.5 x^{2}-134.3 x^{3} ; R^{2}=0.80$

For CDCA: $y=81.58+105.2 x-279.12 \hat{x}+124 x^{3} ; R^{2}=0.74$

For $C A: y=17.72+1.905 x-20.25 x^{2}+8.663 x^{3} ; \mathbb{R}^{2}=0.55$

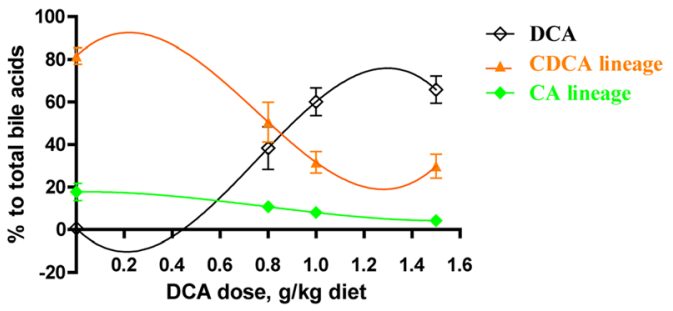

Fig. 6 DCA modulated NE-induced bile acid reduction. Cohorts of 16 broiler chicks were fed basal and DCA supplemented diets (0.8, 1.0 and 1.5 $\mathrm{g} / \mathrm{kg}$ ) and infected as in Fig. 1. a Total bile acids quantification in NE and DCA treated birds. b Individual bile acids quantification in the birds. $\mathbf{c}$ Relative composition of bile acid in the birds. $\mathbf{d}$ Fitting curve for DCA, CDCA lineage (T/GCDCA, CDCA), and CA lineage (T/GCA, CA) in response to different doses of DCA. All graphs showed mean \pm SEM. Results are representative of 3 independent experiments 
was not the optimal dose against NE on body weight gain loss. The lowest DCA dose against subclinical NE-induced body weight gain loss was $0.68 \mathrm{~g} / \mathrm{kg}$ feed, suggesting that a DCA dose lower than $0.68 \mathrm{~g} / \mathrm{kg}$ feed would result in less body weight gain compared to subclinical NE birds. The optimal DCA against subclinical NE on body weight would be $1.262 \mathrm{~g} / \mathrm{kg}$ feed. Future work is needed to dissect which factors contribute to the minimal and optimal doses of DCA against subclinical NE.

The intestinal tract of NE birds often showed a ballooned shape filled with a rotten egg smelling gas, which is the result of an overgrowth of $C$. perfringens [5]. DCA as low as $50 \mathrm{nmol} / \mathrm{L}$ inhibits $C$. perfringens in vitro growth in tryptic soy broth [9]. Consistently, DCA reduced C. perfringens intestinal luminal colonization levels in this subclinical NE. This result is different from our previous DCA against acute NE [9], where DCA doesn't significantly reduce $C$. perfringens luminal colonization. Bile acids/salts are often reported to be broad antimicrobial agents [38]. Recent advancement indicates that specific bile acids inhibit respective bacterial growth. For example, secondary bile acid DCA, but not primary bile acid $\mathrm{CA}$, reduces $C$. difficile in vitro growth [25]. Interestingly, despite the presence of C. perfringens in all groups of birds by PCR assay, C. perfringens was surprisingly barely detected in both uninfected and NE birds fed $1.5 \mathrm{~g} / \mathrm{kg}$ DCA using FISH. The different results between FISH and PCR may be related to the nonspecific DNA amplification by PCR assay, whereas the FISH assay is more specific and reliable. Notably, rod- and spore-shape C. perfringens were easily visualized in ileal lumen and tissue which suggests that $C$. perfringens might experience sporulation and produce enterotoxin (CPE) during this subclinical NE. CPE is implicated in food borne diseases [15] but is rarely investigated in chicken NE. Future work on revealing the role of $\mathrm{CPE}$ on $\mathrm{NE}$ is greatly needed.

The hallmark of acute NE at cellular level is the severe small intestinal inflammation and necrosis in lower jejunum and upper ileum of chickens [9]. Intestinal tissue in the subclinical NE birds of this experiment showed less extended inflammation with shortening villi, crypt hyperplasia and immune cell infiltration. The intestinal inflammation was consistent with an increased expression of proinflammatory mediators of Infy, Mmp9, Il-17a, Il-22, and Il-23. DCA was able to prevent the increased inflammatory mediators. Consistent with the previous report of DCA against acute NE on intestinal inflammation [9], a high dose of DCA at $1.5 \mathrm{~g} / \mathrm{kg}$ feed attenuated NE-induced intestinal inflammation. Furthermore, DCA attenuated intestinal inflammation of histopathology in a dose dependent manner after the construction of the 3rd order polynomial fitting model using dietary DCA levels and an intestinal histopathological score in Fig. 1c. The lowest DCA to reduce a subclinical NE score was at $0.5682 \mathrm{~g} / \mathrm{kg}$, while DCA at $1.359 \mathrm{~g} / \mathrm{kg}$ was the optimal dose to attenuate the NE.
Further work is needed to understand the relationship between DCA doses and NE-induced intestinal inflammation.

Finally, subclinical NE birds with mild diarrhea apparently experienced $67 \%$ less total bile acids in ileal content compared to uninfected birds, whereas NE birds had 28\% less body weight gain loss compared to uninfected birds. The inflammatory bowel disease patients, especially those affecting the distal ileum, often show bile acid malabsorption [39, 40]. Interestingly, the expression of the apical sodium/BA cotransporting polypeptide responsible for ileal BA reabsorption is reduced in ileal biopsies from patients with Crohn's disease free of any signs of inflammation [41]. Although the birds in this experiment had a subclinical NE with no obvious diarrhea present, the loss of bile acids in excrete from enterohepatic cycle in the birds might be one of the factors. In the experiment, dietary DCA was able to compensate the loss of total bile acid reduction in association with reduced $\mathrm{NE}$ on body weight gain loss, intestinal histopathology and inflammation. Bile acid synthesis in liver is regulated by the enterohepatic circulation, but how the impact of individual bile acid on bile acid pool is largely unclear [42]. Cholate is an important negative regulator of bile acid synthesis through the nuclear receptor farnesoid $\mathrm{X}$ receptor and sterol $12 \alpha$-hydroxylase gene (Cyp8b1) in mice [43]. It remains unclear whether the protection from dietary DCA comes from actions to specifically antagonize C. perfringens or just to restore total intestinal bile acid pool. Following up research is much needed to clarify the underlying mechanism.

\section{Conclusion}

The data presented in this study reveals that the microbial metabolic product secondary bile acid DCA attenuates NE by reducing $C$. perfringens colonization, host inflammatory response and bile acid reduction in a dose-dependent manner. These findings highlight the importance of revealing the molecular relationship between infectious pathogen, bile acids and NE resistance/ susceptibility. Discoveries presented here could be applied to control NE and other intestinal diseases targeting microbiome and host response.

\section{Supplementary information}

Supplementary information accompanies this paper at https://doi.org/10. 1186/s40104-020-00441-6.

\footnotetext{
Additional file 1: Supplement Figure 1. Subclinical necrotic enteritis infection reduces feed intake during NE phase. Feed intake of d 23-26 was compared among uninfected, E. maxima and NE groups. The graph showed mean \pm SEM. Results are representative of 3 independent experiments
}

\section{Abbreviations}

BW: Body weight; CA: Cholic acid; CDCA: Chenodeoxycholic acids; CFU: Colony forming unit; DCA: Deoxycholic acid; FISH: Fluorescence in situ hybridization; GCA: Glycol-cholic acids; GCDCA: Glycol-chenodeoxycholic 
acids; LCA: Lithocholic acid; NE: Necrotic enteritis; TCA: Tauro-cholic acids; TCDCA: Tauro-chenodeoxycholic acids; TSB: Tryptic Soy Broth;

TUNEL: Terminal deoxynucleotidyl transferase dUTP nick end labeling assay; UDCA: Ursodeoxycholic acid

\section{Acknowledgements}

The authors would like to thank Dr. Juan D. Latorre for providing E. maxima and C. perfringens, Mr. P. L. Matsler for helping our histology slides and the support from other staffs at Poultry Health Laboratory and Feed Mill in Department of Poultry Science at University of Arkansas, Fayetteville. We also thank Statewide Mass Spectrometry Facility at University of Arkansas at Fayetteville for providing help with targeted metabolomics study.

\section{Authors' contribution}

M.B. and X.S. designed the experiments. M.B., X.S., and Y. F. wrote the manuscript with input from co-authors. M.B. performed animal and in vitro experiments and most analysis with the help of co-authors. The authors read and approved the final manuscript.

\section{Funding}

This research was supported grants of Arkansas Biosciences Institute, USDA National Institute of Food and Agriculture (NIFA) Hatch project 1012366, USDA NIFA Hatch/Multi State project 1018699, USDA NIFA project 201806686 to $X$.

\section{Availability of data and materials}

Not applicable.

\section{Ethics approval}

All procedures with animals received prior approval from the Animal Care and Use Committee of the University of Arkansas.

\section{Consent for publication}

Not applicable

\section{Competing interests}

The authors declare that they have no competing interests.

\section{Author details}

${ }^{1}$ Center of Excellence for Poultry Science, University of Arkansas, $1260 \mathrm{~W}$ Maple St. O409, Fayetteville, AR 72701, USA. ${ }^{2}$ CEMB, University of Arkansas, Fayetteville, AR 72701, USA. ${ }^{3}$ Department of Chemistry, University of Arkansas, Fayetteville, AR 72701, USA.

\section{Received: 1 December 2019 Accepted: 24 February 2020}

\section{Published online: 13 March 2020}

\section{References}

1. Caly DL, D'Inca R, Auclair E, Drider D. Alternatives to antibiotics to prevent necrotic enteritis in broiler chickens: a Microbiologist's perspective. Front Microbiol. 2015;6:1336

2. Diarra MS, Malouin F. Antibiotics in Canadian poultry productions and anticipated alternatives. Front Microbiol. 2014;5:282.

3. Silva RO, Salvarani FM, Assis RA, Martins NR, Pires PS, Lobato FC. Antimicrobial susceptibility of Clostridium perfringens strains isolated from broiler chickens. Braz J Microbiol. 2009;40(2):262-4.

4. Wade B, Keyburn A. The true cost of necrotic enteritis. Poultry World. 2015; 31:16-7.

5. Timbermont L, Haesebrouck F, Ducatelle R, Van Immerseel F. Necrotic enteritis in broilers: an updated review on the pathogenesis. Avian Pathol. 2011;40(4):341-7.

6. Latorre JD, Adhikari B, Park SH, Teague KD, Graham LE, Mahaffey BD, et al. Evaluation of the epithelial barrier function and lleal microbiome in an established necrotic enteritis challenge model in broiler chickens. Front Vet Sci. 2018:5:199.

7. Long JR, Pettit JR, Barnum DA. Necrotic enteritis in broiler chickens. II. Pathology and proposed pathogenesis. Can J Comp Med. 1974;38(4):467-74

8. Al-Sheikhly F, Truscott RB. The pathology of necrotic enteritis of chickens following infusion of broth cultures of Clostridium perfringens into the duodenum. Avian Dis. 1977;21(2):230-40.
9. Wang H, Latorre JD, Bansal M, Abraha M, Al-Rubaye B, Tellez-Isaias G, et al Microbial metabolite deoxycholic acid controls Clostridium perfringensinduced chicken necrotic enteritis through attenuating inflammatory cyclooxygenase signaling. Sci Rep. 2019;9(1):14541.

10. Prescott JF, Parreira VR, Mehdizadeh Gohari I, Lepp D, Gong J. The pathogenesis of necrotic enteritis in chickens: what we know and what we need to know: a review. Avian Pathol. 2016;45(3):288-94.

11. Craven SE. Colonization of the intestinal tract by Clostridium perfringens and fecal shedding in diet-stressed and unstressed broiler chickens. Poult Sci. 2000;79(6):843-9.

12. Pedersen K, Bjerrum L, Nauerby B, Madsen M. Experimental infections with rifampicin-resistant Clostridium perfringens strains in broiler chickens using isolator facilities. Avian Pathol. 2003;32(4):403-11.

13. Kaldhusdal M, Hofshagen M, Lovland A, Langstrand H, Redhead K. Necrotic enteritis challenge models with broiler chickens raised on litter: evaluation of preconditions, Clostridium perfringens strains and outcome variables. FEMS Immunol Med Microbiol. 1999;24(3):337-43.

14. Williams RB. Intercurrent coccidiosis and necrotic enteritis of chickens: rational, integrated disease management by maintenance of gut integrity. Avian Pathol. 2005;34(3):159-80.

15. Navarro MA, McClane BA, Uzal FA. Mechanisms of Action and Cell Death Associated with Clostridium perfringens Toxins. Toxins (Basel). 2018;10:212.

16. Keyburn AL, Boyce JD, Vaz P, Bannam TL, Ford ME, Parker D, et al. NetB, a new toxin that is associated with avian necrotic enteritis caused by Clostridium perfringens. PLoS Pathog. 2008;4(2):e26.

17. Martin TG, Smyth JA. Prevalence of netB among some clinical isolates of Clostridium perfringens from animals in the United States. Vet Microbiol. 2009:136(1-2):202-5.

18. Yang WY, Chou CH, Wang C. Characterization of toxin genes and quantitative analysis of netB in necrotic enteritis (NE)-producing and nonNE-producing Clostridium perfringens isolated from chickens. Anaerobe. 2018:54:115-20

19. Caricilli AM, Castoldi A, Camara NO. Intestinal barrier: a gentlemen's agreement between microbiota and immunity. World J Gastrointest Pathophysiol. 2014;5(1):18-32.

20. Subramanian S, Huq S, Yatsunenko T, Haque R, Mahfuz M, Alam MA, et al. Persistent gut microbiota immaturity in malnourished Bangladeshi children. Nature. 2014:510:417-21.

21. Belkaid $Y$, Hand TW. Role of the microbiota in immunity and inflammation. Cell. 2014;157(1):121-41.

22. Sun $X$, Jia Z. Microbiome modulates intestinal homeostasis against inflammatory diseases. Vet Immunol Immunopathol. 2018;205:97-105.

23. Antonissen $G$, Eeckhaut V, Van Driessche K, Onrust L, Haesebrouck F, Ducatelle $R$, et al. Microbial shifts associated with necrotic enteritis. Avian Pathol. 2016;45(3):308-12.

24. Silverman MS, Davis I, Pillai DR. Success of self-administered home fecal transplantation for chronic Clostridium difficile infection. Clin Gastroenterol Hepatol. 2010;8(5):471-3

25. Buffie CG, Bucci V, Stein RR, McKenney PT, Ling L, Gobourne A, et al. Precision microbiome reconstitution restores bile acid mediated resistance to Clostridium difficile. Nature. 2015:517(7533):205-8.

26. Ridlon JM, Kang DJ, Hylemon PB. Bile salt biotransformations by human intestinal bacteria. J Lipid Res. 2006:47(2):241-59.

27. Ma H, Patti ME. Bile acids, obesity, and the metabolic syndrome. Best Pract Res Clin Gastroenterol. 2014;28(4):573-83.

28. Bernstein C, Holubec H, Bhattacharyya AK, Nguyen H, Payne CM, Zaitlin B, et al. Carcinogenicity of deoxycholate, a secondary bile acid. Arch Toxicol. 2011;85(8):863-71.

29. Yano JM, Yu K, Donaldson GP, Shastri GG, Ann P, Ma L, et al. Indigenous bacteria from the gut microbiota regulate host serotonin biosynthesis. Cell. 2015;161(2):264-76.

30. Sun X, Winglee K, Gharaibeh RZ, Gauthier J, He Z, Tripathi P, et al. Microbiota-derived metabolic factors reduce Campylobacteriosis in mice. Gastroenterology. 2018;154(6):1751-63 e2.

31. Alrubaye B, Abraha M, Almansour A, Bansal M, Wang H, Kwon YM, et al. Microbial metabolite deoxycholic acid shapes microbiota against Campylobacter jejuni chicken colonization. PLoS One. 2019;14(7):e0214705.

32. Shivaramaiah S, Wolfenden RE, Barta JR, Morgan MJ, Wolfenden AD, Hargis $\mathrm{BM}$, et al. The role of an early Salmonella Typhimurium infection as a predisposing factor for necrotic enteritis in a laboratory challenge model. Avian Dis. 2011;55(2):319-23. 
33. McReynolds JL, Byrd JA, Anderson RC, Moore RW, Edrington TS, Genovese $\mathrm{KJ}$, et al. Evaluation of immunosuppressants and dietary mechanisms in an experimental disease model for necrotic enteritis. Poult Sci. 2004;83(12): 1948-52.

34. Sun X, Threadgill D, Jobin C. Campylobacter jejuni induces colitis through activation of mammalian target of rapamycin signaling. Gastroenterology. 2012;142(1):86-95 e5.

35. Yuan JS, Reed A, Chen F, Stewart CN Jr. Statistical analysis of real-time PCR data. BMC Bioinformatics. 2006;7:85.

36. Diaz Perez A, Kougl K, Vasicek TW, Liyanage R, Lay J, Stenken JA. Microdialysis sampling of quorum sensing Homoserine lactones during biofilm formation. Anal Chem. 2019;91(6):3964-70.

37. Conway DP, McKenzie ME, Dayton AD. Relationship of coccidial lesion scores and weight gain in infections of Eimeria acervulina, E. maxima and $E$. tenella in broilers. Avian Pathol. 1990;19(3):489-96.

38. Urdaneta V, Casadesus J. Interactions between Bacteria and Bile Salts in the Gastrointestinal and Hepatobiliary Tracts. Front Med (Lausanne). 2017;4:163.

39. Gothe F, Beigel F, Rust C, Hajji M, Koletzko S, Freudenberg F. Bile acid malabsorption assessed by 7 alpha-hydroxy-4-cholesten-3-one in pediatric inflammatory bowel disease: correlation to clinical and laboratory findings. J Crohns Colitis. 2014;8(9):1072-8.

40. Vitek L. Bile acid malabsorption in inflammatory bowel disease. Inflamm Bowel Dis. 2015;21(2):476-83.

41. Jung D, Fantin AC, Scheurer U, Fried M, Kullak-Ublick GA. Human ileal bile acid transporter gene ASBT (SLC10A2) is transactivated by the glucocorticoid receptor. Gut. 2004:53(1):78-84.

42. Chiang JY. Negative feedback regulation of bile acid metabolism: impact on liver metabolism and diseases. Hepatology. 2015;62(4):1315-7.

43. Li-Hawkins J, Gafvels M, Olin M, Lund EG, Andersson U, Schuster G, et al. Cholic acid mediates negative feedback regulation of bile acid synthesis in mice. J Clin Invest. 2002;110(8):1191-200.

Ready to submit your research? Choose BMC and benefit from:

- fast, convenient online submission

- thorough peer review by experienced researchers in your field

- rapid publication on acceptance

- support for research data, including large and complex data types

- gold Open Access which fosters wider collaboration and increased citations

- maximum visibility for your research: over $100 \mathrm{M}$ website views per year

At $\mathrm{BMC}$, research is always in progress.

Learn more biomedcentral.com/submissions 